\title{
Variantes moleculares en el gen PARK2 en pacientes colombianos con enfermedad de Parkinson. Estudio piloto entre el 2013 y 2014
}

\author{
Sergio Andrés Castañeda-Garzón* \\ Luisa Fernanda Urrego-Duque ${ }^{* *}$ \\ Magda Carolina Sánchez-Corredor***
}

\begin{abstract}
*Bacteriólogo. Especialista en Epidemiología. Universidad del Rosario. Facultad de Ciencias Naturales y Matemáticas. Bogotá. Colombia. **Bacterióloga. Magister en Genética Humana. Universidad del Rosario. Facultad de Ciencias Naturales y Matemáticas. Bogotá. Colombia. ***Química. Magister en Genética Humana. Universidad del Rosario. Facultad de Ciencias Naturales y Matemáticas. Bogotá. Colombia. Correspondencia: Sra. Magda Carolina Sánchez Corredor. Dirección: Carrera 26 \#63B-48. Barrio 7 de Agosto. Bogotá D.C. Colombia. Correo electrónico: magda.sanchez@urosario.edu.co
\end{abstract}

\section{RESUMEN}

Introducción: la enfermedad de Parkinson se caracteriza por la degeneración y pérdida de las neuronas dopaminérgicas en el cerebro. Existen factores genéticos involucrados en su desarrollo, en su forma de inicio temprano como el gen PARK2, codificante de la parkina, una $\mathrm{E} 3$ ubiquitín ligasa, lo que hace que en caso de mutaciones pierda su capacidad reguladora de degradación de proteínas causando estrés y muerte celular. Objetivo: determinar la presencia de cambios moleculares en los exones 3, 4, y 5 de PARK2 en un grupo de 29 pacientes y 21 controles colombianos con enfermedad de Parkinson de inicio temprano o con antecedentes familiares de ella y la posible correlación con las manifestaciones clínicas de los pacientes. Materiales y métodos: estudio descriptivo observacional (entre junio de 2013 y noviembre de 2014) en donde se realizó extracción de ADN de sangre total y se utilizó la técnica de PCR para cada uno de los exones. Finalmente se procedió a la secuenciación automática, análisis de las secuencias con el software Sequencher y comparación con información de bases de datos. Resultados: se identificó una variante en estado homocigoto (Ala46Thr) en el exón 4 en un paciente no reportada anteriormente, posiblemente no patogénica y la variante Ser167Asn en estado heterocigoto en el mismo exón en otro paciente, considerada patogénica y reportada con anterioridad en poblaciones asiática y europea. No se identificaron variantes en los controles. Conclusiones: los cambios no descritos antes en la población colombiana, -Ala46Thr y Ser167Asn-, fueron identificados en el grupo de pacientes. MÉD.UIS. 2017;30(3):31-8.

Palabras clave: Enfermedad de Parkinson. Mutación. Neuronas.

\section{Molecular variants in gene PARK2 in Colombian patients with Parkinson's disease. Pilot study between 2013 and 2014}

\section{ABSTRACT}

Introduction: parkinson's disease is characterized by the degeneration and loss of dopaminergic neurons in the brain. There are genetic factors involved in its development in its early onset form, such as the PARK2 gene encoding the parkin, an E3 ubiquitin ligase, which in the case of mutations loses its ability to regulate protein degradation causing stress and cell death. Objective: to determine the presence of molecular changes in PARK2 exons 3, 4 and 5 in a group of 29 patients and 21 colombian controls with early onset Parkinson's disease or a family history of Parkinson's disease and the possible correlation with clinical manifestations from the patients. Materials and methods: observational descriptive study (between june of 2013 and november of 2014) where DNA extraction of whole blood was performed and the PCR technique was used for each of the exons. Finally, we proceeded to the automatic sequencing, analysis of the sequences with the Sequencher software and comparison with information of databases. Results: a homozygous variant (Ala46Thr) in exon 4 was identified in one patient not previously reported, possibly nonpathogenic and the Ser167Asn variant in heterozygous state in the same exon in another patient, considered pathogenic and previously reported in populations Asian and European. No variants were identified in the controls. Conclusions: changes not previously described in the colombian population, -Ala46Thr and Ser167Asn-, were identified in the group of patients and not in the controls. MÉD.UIS. 2017;30(3):31-8.

Keywords: Parkinson's disease. Mutation. Neurons.

Artículo recibido el 29 de diciembre de 2016 y aceptado para publicación el 23 de mayo de 2017 
¿Cómo citar este artículo?: Castañeda-Garzón SA, Urrego-Duque LF, Sánchez-Corredor MG. Variantes moleculares en el gen PARK2 en pacientes colombianos con enfermedad de Parkinson. Estudio piloto entre el 2013 y 2014. MÉD.UIS. 2017;30(3):31-8.

\section{INTRODUCCIÓN}

Después de la enfermedad de Alzheimer, la Enfermedad de Parkinson (EP) es el segundo desorden neurodegenerativo más frecuente y la causa más común de parkinsonismo ${ }^{1,2}$, con una prevalencia en la población general de $0,3 \%$ y en personas mayores de 65 años de hasta $3 \%$. Aunque afecta indistintamente a cualquier grupo étnico y género, su prevalencia es mayor en los varones y en los individuos hispanos con respecto a los de origen afroamericano ${ }^{3}$.

La sintomatología asociada incluye alteraciones del movimiento tales como bradiquinesia, temblor e inestabilidad postural ${ }^{4}$, causadas por la deficiencia de dopamina resultante de la degeneración de las neuronas dopaminérgicas en diversas regiones del cerebro, como la sustancia nigra, el locus cerúleo y los ganglios basales ${ }^{1,5}$. La EP es una enfermedad compleja y multifactorial, en la que convergen factores genéticos y ambientales como infecciones por ciertos virus o exposición a neurotoxinas ${ }^{6}$. Hasta la fecha han sido identificadas mutaciones en más de diez genes implicados en los mecanismos patogénicos de la EP, bien sea como causantes o como genes de susceptibilidad para la enfermedad (Ver Tabla 1) $)^{7-10}$. Uno de estos genes, PARK2 (OMIM 602544), localizado en el cromosoma 6q25.2-q27, codifica la parkina, proteína de 465 aminoácidos y un peso molecular de 51,652 $\mathrm{D}^{11}$. La función como E3 ubiquitín ligasa de la parkina es decisoria para la remoción proteasomal de las proteínas ubiquitinadas ${ }^{11,12}$. En presencia de mutaciones en PARK2, las proteínas mal plegadas se acumulan en el citoplasma y se tornan tóxicas para las neuronas. En consecuencia, pueden ocurrir alteraciones funcionales en las vesículas sinápticas, lo que provoca un almacenamiento defectuoso de la dopamina, seguido de estrés oxidativo y la muerte celular'13. Más de 100 mutaciones en PARK2 han sido reportadas en poblaciones europeas y asiáticas, principalmente en los exones 2, 3, 4, 5 y $7^{4,14-17}$.

Tabla 1. Loci y genes asociados con enfermedad de Parkinson

\begin{tabular}{|c|c|c|c|c|c|}
\hline Locus & $\begin{array}{l}\text { Localización } \\
\text { cromosómica }\end{array}$ & $\begin{array}{l}\text { Patrón de } \\
\text { herencia }\end{array}$ & Proteína & Presunta función & Referencia \\
\hline PARK 1 & $4 q 21$ & $A D$ & a-Sinucleína & NC & $\begin{array}{l}\text { Polymeropolous y cols. } \\
1997^{26}\end{array}$ \\
\hline PARK 2 & $6 q 25.2-27$ & $A R$ & parkina & E3 ubiquitin Ligasa & Kitada y cols. $1998^{20}$ \\
\hline PARK 3 & $2 p 13$ & $A D$ & NC & NC & Gasser T. $1998^{27}$ \\
\hline PARK 5 & $4 p 14$ & $A D$ & UCH-L1 & $\begin{array}{c}\text { Ubiquitna C-terminal } \\
\text { Hidrolasa }\end{array}$ & Leroy y cols. $1998^{28}$ \\
\hline PARK 6 & $1 p 36$ & $A R$ & PINK1 & $\begin{array}{l}\text { Proteín-cinasa } \\
\text { Mitocondrial }\end{array}$ & Valente y cols. $2001^{29}$ \\
\hline PARK 7 & $1 \mathrm{p} 36$ & $A R$ & DJ-1 & $\begin{array}{c}\text { Chaperona respuesta a } \\
\text { estréss oxidativo }\end{array}$ & Van Duijin y cols. $2001^{30}$ \\
\hline PARK 8 & $12 \mathrm{p} 11.2$ & $A D$ & Dardarina & LRRK2 Protein-cinasa & Paísan-Ruiz y cols. $2004^{31}$ \\
\hline PARK 10 & 1 p32 & $A D$ & NC & NC & Hicks y cols. $2002^{32}$ \\
\hline PARK 11 & $2 q 36$ & $A D$ & NC & NC & Pankratz y cols. $2001^{33}$ \\
\hline
\end{tabular}

Fuente: Modificado de Morris HR $^{10}$. Muestra los loci relacionados con enfermedad de parkinson familiar. AD:autosómico dominante, AR:autosómico recesivo. NC:no conocida. PARK4 fue excluido ya que corresponde a la triplicación del gen de PARK1. PARK9 fue excluido ya que se encontró que no era un locus de Enfermedad de Parkinson. 
En la EP de Inicio Temprano (EPIT), de aparición antes de los 50 años $^{11}$, se han identificado diversas mutaciones en genes asociados con el desarrollo de la enfermedad 6 , entre los cuales también se encuentra PARK $2^{18}$. Así, la presencia de mutaciones en este gen ha sido informada en un $50 \%$ de los casos familiares de EPIT y en un 10\% de los casos esporádicos de EP en pacientes europeos ${ }^{6,11,15}$, mientras que en pacientes colombianos con EPIT, solo ha sido reportada la mutación C255delA en el exón 2 de PARK2, la cual fue identificada en cuatro de los diez hijos de una gran familia del sur de Colombia, con una historia de consanguinidad entre los padres ${ }^{14}$.

En su forma de inicio temprano, el estudio de la EP presenta retos importantes pues, aunque se ha establecido que posee un componente genético, éste no ha sido completamente dilucidado. El presente estudio tiene como objetivo determinar la presencia de cambios moleculares en los exones 3, 4, y 5 de PARK2 en un grupo de personas colombianas con EPIT o con antecedentes familiares de ella y la posible correlación con los hallazgos clínicos de los pacientes.

\section{Materiales y mÉtodos}

\section{DISEÑO DEL ESTUDIO}

Corresponde a un estudio descriptivo observacional de tipo corte transversal, realizado entre junio de 2013 y noviembre de 2014, la muestra poblacional estuvo constituida por 29 personas de ambos sexos diagnosticadas con EP que cursan con EPIT o con antecedentes familiares de esta enfermedad, contactados a través de la Liga de Parkinson de Bogotá, Colombia y 21 controles sanos sin antecedentes de enfermedades neurológicas quienes previo consentimiento informado y de acuerdo con la Declaración de Helsinki y el Reporte de Belmont, fueron incorporados en el estudio.

\section{POBLACIÓN Y CRITERIOS DE SELECCIÓN}

Los criterios de inclusión fueron individuos diagnosticados con EP de inicio anterior a los 50 años de edad (diagnóstico de la enfermedad verificado con la historia clínica del paciente) e individuos que aceptaran su participación de forma voluntaria en el estudio. La totalidad de los pacientes presentaba los tres hallazgos considerados criterios necesarios para que su padecimiento se clasificara como una EP clásica (bradiquinesia, temblor en reposo e inestabilidad postural), en algunos casos más marcados de acuerdo con el tiempo de progreso de la enfermedad. Dos de los 29 casos presentaba una herencia autosómico recesiva y los otros 27 fueron casos esporádicos en la familia. En el estudio también fueron incluidos individuos sanos que confromaron el grupo control, quienes cumplían con los siguientes criterios de inclusión: edad entre 18 y 50 años e individuos sin ningún antecedente familiar de EP o alguna otra enfermedad neurodegenerativa. Como criterios de exclusión se aplicaron el diagnóstico de alguna enfermedad neurodegenerativa como Alzheimer y sujetos sin diagnóstico diferencial de Parkinson, o en aquellos en los que no se haya aclarado la patología específica, antes de los 50 años de edad.

\section{VARIABLES ANALIZADAS}

Se analizaron variables dependientes como mutación en los exones 3, 4 y 5 del gen PARK2, variable independiente, la EPIT y como variables de caracterización la edad (años) y sexo (masculino/ femenino) - (Ver Tabla 2).

\section{EXTRACCIÓN DE MUESTRA Y PROCESAMIENTO DE LA INFORMACIÓN}

El ADN fue extraído a partir de muestras de sangre periférica con el equipo comercial de extracción salina CorpoGen DNA $2000^{\circledR}$ BM-001. De cada paciente se tomaron dos tubos con anticoagulante EDTA que se almacenaron en refrigeración hasta el momento de ser procesados, una vez realizado el proceso de extracción, se procedió a un chequeo en geles de agarosa al uno por ciento para verificación de la misma. Se hizo una evaluación de la concentración de cada muestra de ADN mediante espectrofotometría, se efectuó la amplificación (PCR) previamente estandarizada con muestras control de individuos sanos, de los exones 3, 4 y 5 del gen PARK2 en termociclador BIORAD T100, con el equipo Promega $\mathrm{GoTaq}^{T M}$ Flexi DNA Polymerase M8095. Para este efecto, fueron utilizados los siguientes cebadores 0 primers (Ver Tabla 3, 4 y 5) $)^{19,20}$. 
Tabla 2. Tabla Operacional De Variables

\begin{tabular}{|c|c|c|c|c|c|c|}
\hline Grupo de variables & Variable & $\begin{array}{l}\text { Tipo de } \\
\text { variable }\end{array}$ & Nombre & Indicador & Código & $\begin{array}{c}\text { Nivel } \\
\text { de } \\
\text { Medición }\end{array}$ \\
\hline \multirow[b]{2}{*}{ Caracterización } & Edad & Cuantitativa & Edad & Años & Edad en años & De razón \\
\hline & Género & Cualitativo & Sexo & $\%$ & $\begin{array}{l}\text { 1.Femenino o } \\
\text { 2.Masculino }\end{array}$ & Nominal \\
\hline \multirow{3}{*}{ Resultados } & $\begin{array}{c}\text { Mutación en exón } \\
3\end{array}$ & Cualitativa & MUT E3 & $\%$ & $\begin{array}{l}\text { 0. No } \\
\text { 1. Si }\end{array}$ & Nominal \\
\hline & $\begin{array}{c}\text { Mutación en exón } \\
4\end{array}$ & Cualitativa & MUT E4 & $\%$ & $\begin{array}{l}\text { 0. No } \\
\text { 1. Si }\end{array}$ & Nominal \\
\hline & $\begin{array}{c}\text { Mutación en exón } \\
5\end{array}$ & Cualitativa & MUT E5 & $\%$ & $\begin{array}{l}\text { 0. No } \\
\text { 1. Si }\end{array}$ & Nominal \\
\hline
\end{tabular}

Fuente: Autores.

Tabla 3. Primers Utilizados

\begin{tabular}{|l|l|}
\hline \multirow{2}{*}{ Exón 3} & F 5' CTTGCTCCCAAACAGAATT 3' \\
\cline { 2 - 2 } & R 5' AGGCCATGCTCCATGCAGACTGC 3' \\
\hline \multirow{2}{*}{ Exón 4} & F 5' ACAAGCTTTTAAAGAGTTTCTTGT 3' \\
\cline { 2 - 2 } & R 5' AGGCAATGTGTTAGTACACA 3' \\
\hline \multirow{2}{*}{ Exón 5 } & F 5' ACATGTCTTAAGGAGTACATTT 3' \\
\cline { 2 - 2 } & R 5' TCTCTAATTTCCTGGCAAACAGTG 3' \\
\hline
\end{tabular}

Fuente: Autores.

Tabla 4. Condiciones De Amplificación

\begin{tabular}{|c|c|c|c|}
\hline Reactivos en ul & EXÓN 3 & EXÓN 4 & EXÓN 5 \\
\hline dNTPs & 1,5 & 1,5 & 2 \\
\hline Buffer & 1,5 & 1,5 & 2 \\
\hline MgCL2 & 2,5 & 2 & 3 \\
\hline Primer F & 1 & 1 & 1 \\
\hline Primer R & 1 & 1 & 1 \\
\hline DNA & 1 & 1 & 2 \\
\hline Taq Polimerasa & 0,1 & 0,1 & 0,1 \\
\hline Agua & 9,4 & 9,9 & 6,9 \\
\hline TOTAL & 18 & 18 & 18 \\
\hline
\end{tabular}

Fuente: Autores.

Posteriormente se realizó un nuevo chequeo en agarosa para comprobar la amplificación. La recolección de las muestras se llevó a cabo por parte de la bacterióloga y en algunos casos la auxiliar de laboratorio, especialista en toma de muestra. La parte experimental en su totalidad fue realizada por un estudiante de especialización en epidemiología que pertenece al grupo de investigación Ciencias Básicas Médicas de la Universidad del Rosario. El trabajo experimental y teórico fue supervisado y dirigido por las docentes.
Una vez obtenidos los productos amplificados, se efectuó secuenciación de los tres exones para cada paciente en ELIM Biopharmaceuticals, Inc. USA. Las secuencias fueron analizadas con el software Sequencher y comparadas con la información contenida en las bases de datos Ensembl y NCBI. La posible asociación de riesgo entre la presencia de cambios y la manifestación de la enfermedad fue estimada mediante el odds ratio mientras que la significancia fue evaluada con el test exacto de Fisher. Los resultados fueron analizados con el Software EPIDAT 3,1 (2006).

\section{Resultados}

Se contó con un total de 50 participantes, dentro de los casos el $41,4 \%$ de las personas con EPIT o con antecedentes familiares fueron hombres y el $58,6 \%$ mujeres (Ver Tabla 6). Los resultados no mostraron cambios en los exones 3 y 5 de gen PARK2. No obstante, en el exón 4 de dos individuos se identificaron dos variantes causantes de alteraciones en la proteína, la primera es la transición G>A (Ver Figura 1) en estado homocigoto que provoca el cambio Ala46Thr en la proteína, pero sin alterar su longitud. Después de efectuar la búsqueda correspondiente en las bases de datos, se estableció que se trata de un cambio no reportado antes en la población colombiana. El segundo es un cambio en estado heterocigoto que ya había sido reportado en otras poblaciones ${ }^{21,22}$, este cambio C.500G >A (Ver Figura 2) provoca a su vez el remplazo Ser167Asn en la proteína ${ }^{21}$, responsable de modificaciones en el tamaño y la estructura de la proteína. Igualmente se identificó en el exón 4 del control $\mathrm{C} 020$ un cambio sinónimo de $\mathrm{G}>\mathrm{A}$ en estado 
SEPTIEMBRE - DICIEMBRE

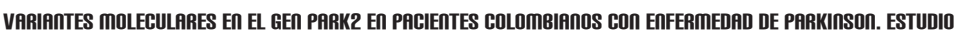

heterocigoto que no representa ninguna alteración en la proteína, por tanto puede tratarse de un polimorfismo que no genera ninguna alteración funcional de la misma y no está aparentemente involucrado en la aparición o desarrollo de la enfermedad (Ver Tabla 7).

Tabla 5. Condiciones Del Termociclador

\begin{tabular}{|c|c|c|c|}
\hline Temperatura $\left({ }^{\circ} \mathbf{C}\right) \mathbf{P C R}$ & Exón 3 (427pb) & Exón 4 (261 pb) & Exón 5 (227pb) \\
\hline Denaturación inicial & $94 \times 10 \mathrm{~min}$. & $94 \times 10 \mathrm{~min}$. & $94 \times 10 \mathrm{~min}$. \\
\hline Denaturación inicial & $94 \times 30 \mathrm{seg}$. & $94 \times 30 \mathrm{seg}$. & $54 \times 30 \mathrm{seg}$. \\
\hline Anillamiento & $57 \times 30 \mathrm{seg}$. & $54 \times 30 \mathrm{seg}$. & $72 \times 45 \mathrm{seg}$. \\
\hline Elongación & $72 \times 45 \mathrm{seg}$. & $72 \times 45 \mathrm{seg}$. & $72 \times 10 \mathrm{~min}$. \\
\hline Elongación Final & $72 \times 10 \mathrm{~min}$. & $72 \times 10 \mathrm{~min}$. & $30 \mathrm{seg}$ \\
\hline \# de Ciclos & $30 \mathrm{X}$ & $30 \mathrm{X}$ & \\
\hline
\end{tabular}

Fuente: Autores.

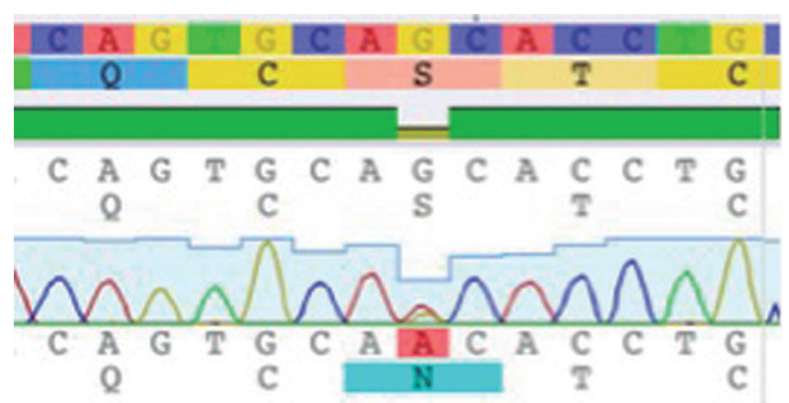

Figura 1. Cambio $\mathrm{G}>\mathrm{A}$ en estado homocigoto, que provoca el cambio en el aminoácido Ala46Thr

Fuente: Autores.

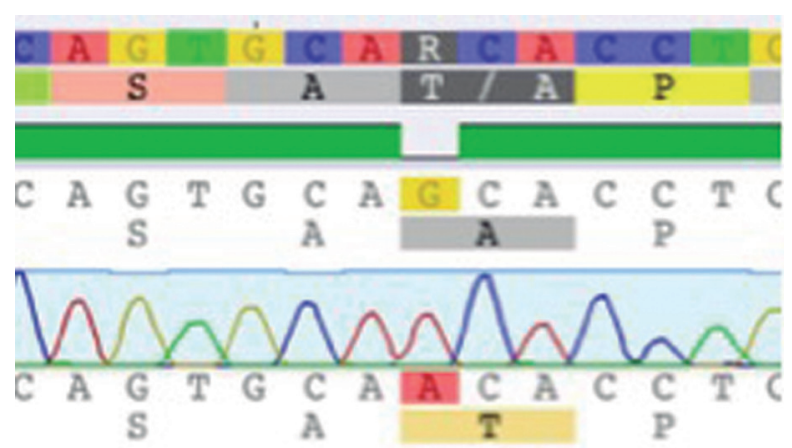

Figura 2. Cambio $\mathrm{G}>\mathrm{A}$ en estado homocigoto, que induce el cambio Ser167Asn en la proteína

Fuente: Autores.

Se realizó una estimación (por tratarse de un estudio transversal) de la posible asociación utilizando el cálculo de OR, y se aplicó un test exacto de Fisher para evaluar significancia con el software EPIDAT 3,1 (2006). El resultado mostró que no existen diferencias estadísticamente significativas en los resultados obtenidos entre los casos y controles evaluados (Ver Tabla 7).
Tabla 6. Datos Demográficos de muestras de pacientes y controles analizadas

\begin{tabular}{|c|c|c|}
\hline Pacientes & Con EPIT & $\begin{array}{c}\text { Con Antecedentes } \\
\text { familiares }\end{array}$ \\
\hline Género & & 2 \\
Femenino & 17 & 0 \\
\hline Masculino & 12 & 2 \\
\hline $\begin{array}{c}\text { Promedio edad de } \\
\text { inicio PD en años }\end{array}$ & 29 & $40+/-8$ \\
\hline \multicolumn{2}{|c|}{$(32-48)$} \\
\hline \multicolumn{2}{|c|}{$44+/-7,6(39-50)$} \\
\hline Género & Controles & 0 \\
\hline Femenino & 12 & 0 \\
\hline Masculino & 9 & 0 \\
\hline Edad en años & 21 & \\
\hline
\end{tabular}

Fuente: Autores.

Tabla 7. Tabla de contingencia $2 \times 2$

\begin{tabular}{|c|c|c|c|}
\hline & Variantes exón & Normal & Total \\
\hline Pacientes EP & 2 & 27 & 29 \\
\hline Controles & 1 & 20 & 21 \\
\hline Total & 3 & 47 & 50 \\
\hline OR & \multicolumn{3}{|c|}{1,48} \\
\hline IC & \multicolumn{3}{|c|}{$95 \%(0,12-17,49)$} \\
\hline p & \multicolumn{3}{|c|}{0,62} \\
\hline
\end{tabular}

Fuente: Autores. 


\section{Discusión}

La EPIT es una patología con una clara influencia genética, sin embargo, los mecanismos y las alteraciones moleculares asociadas no han sido completamente dilucidados. Se ha sugerido que las mutaciones en el gen PARK2 llevan a la pérdida de función de la parkina porque reducen su capacidad de regular la degradación de sustratos. En este sentido, se ha observado que bajo condiciones normales la parkina liga una forma O-glicosilada de la a-sinucleína, proteína codificada por gen PARK1, también clave en la etiología de la EP para llevarla a remoción en el proteasoma. Así, la pérdida de función de la parkina está relacionada con la acumulación de $a$-sinucleína en el citoplasma y en su agregación anormal en los cuerpos de Lewy presentes en las neuronas de la sustancia nigra de los pacientes con $E P^{13}$. La parkina también regula la degradación de proteínas que participan en el ciclo celular como la ciclina E, de modo que cuando el gen PARK2 está mutado la ciclina E se acumula, lo que lleva a la muerte neuronal por apoptosis ${ }^{22}$.

Este estudio permitió identificar dos variantes en la secuencia del gen PARK2. Una de ellas fue la variante en estado homocigoto $G>A$ en el exón 4 que genera la modificación Ala46Thr y no altera la longitud de la proteína. Esta variación ha sido reportada en una población africana (Nigeria) en uno de 57 pacientes con $\mathrm{EP}^{23}$. En razón de que esta variante no ha sido asociada con las manifestaciones fenotípicas patológicas de la enfermedad, es posible que se trate de un polimorfismo que no genera efectos fisiopatológicos sobre su desarrollo o sobre el riesgo de padecerla. No obstante, el hecho de que se encuentre en estado homocigoto y que dé origen a modificaciones en la secuencia de aminoácidos sin alterar la longitud de la proteína, se puede considerar como objetivo de estudio en futuras investigaciones relacionadas con EP. Otro de los integrantes del grupo de estudio con antecedentes familiares de la enfermedad, presentó un cambio en estado heterocigoto $\mathrm{G}>\mathrm{A}$ causante del cambio Ser167Asn, la que aunque ya había sido identificada en otras poblaciones ${ }^{21,22,24}$, no había sido descrita en Colombia. Sanyal y cols., en el 2011 identificaron cinco polimorfismos del gen PARK2 en 1000 individuos sanos procedentes de diez poblaciones indias diferentes, identificando la variante Ser167Asn con la menor frecuencia alélica del 3\% en Gallong y la mayor frecuencia alélica identificada fue del $17 \%$ en la población de Kathakur ${ }^{21}$. Zhang y cols., durante el mismo año realizaron un estudio de la variante Ser167Asn del gen PARK2 con la participación de 2280 casos de pacientes con EP (1065 caucásicos, 1086 asiáticos, 72 americanos latinos y 57 negros africanos) y 2459 controles, en el cual concluyen que esta variante no se asocia con el desarrollo de la EP'22. Lo expuesto por estos autores está en desacuerdo con lo propuesto en 2003 por Peng y col, quienes realizaron un estudio en 25 pacientes con EPIT, 91 con EP de inicio tardía y 124 controles en una población del suroeste de China, en la cual proponen de acuerdo con los resultados obtenidos que la variante Ser167Asn incremente el riesgo de desarrollar EP24.

Se ha sugerido que este cambio en el aminoácido genera una importante modificación estructural y funcional de la proteína, relacionada con alteraciones en su tamaño y plegamiento, motivo por el cual puede estar directamente involucrado en la aparición y desarrollo de la enfermedad. Aunque el efecto de esta mutación sobre la fisiopatología de la enfermedad no ha sido aclarado, se ha propuesto que altera el acoplamiento entre el dominio de unión al sustrato de la parkina y el sustrato protéico blanco $^{24}$. Esta variante podría ser tenida en cuenta como un posible biomarcador de susceptibilidad al desarrollo de la enfermedad. En ambos casos (Ala46Thr y Ser167Asn) los pacientes presentaron un inicio de la enfermedad antes de los 50 años y se observan hallazgos clínicos clásicos de la enfermedad que incluyen bradiquinesia, temblor en reposo e inestabilidad postural, adicionalmente, en el comienzo de la enfermedad respondieron en forma favorable al uso de la levodopa como tratamiento.

Con miras de confirmar la posible relación entre la variante y la enfermedad se hace indispensable una revisión en al menos 100 controles sanos y estudios adicionales de modelos de la proteína con el fin de confirmar la patogenicidad de este cambio y así poder afirmar con certeza si se trata de una mutación patológica causante de EP o si es solo un polimorfismo, lo que debería ser uno de los pasos a seguir en futuras investigaciones, dado que estudios anteriores sugieren que mutaciones puntuales en el gen PARK2 no son exclusivas de pacientes EP ${ }^{25}$.

El análisis estadístico no mostró asociación significativa de riesgo entre la presencia de variantes y la manifestación de la enfermedad ( $O R=1,48$; IC 95\%; $p=0,62$, prueba exacta de Fisher). Sin embargo, son necesarios estudios de expresión asociados a las variantes identificadas con el objetivo de determinar 
si nuestra población mestiza se comporta de igual manera que otras poblaciones en las cuales se han identificado. De igual manera, es importante establecer si hay diferencias en cuanto al sexo, la edad de inicio y los hallazgos clínicos de otros estudios con los reportados en este trabajo, resultados que no se reportan actualmente. La identificación de estas mutaciones permitirá a partir de estudios epidemiológicos, de asociación y causalidad, facilitar la implementación de paneles de detección de estas mutaciones que permita realizar un diagnóstico temprano de la enfermedad y posibles nuevas dianas terapéuticas, en caso de obtener resultados positivos en futuras investigaciones.

En virtud del tamaño de muestra y de la baja prevalencia de la EP, este estudio tuvo el carácter de piloto, por lo que no es comparable con estudios realizados en otras poblaciones con un tamaño de muestra semejante. Vale la pena resaltar que las variantes (Ala46Thr y Ser167Asn) halladas en esta investigación no han sido reportadas en estudios previos realizados en población colombiana y que sólo se ha reportado previamente la mutación en estado homocigoto c.255delA, en el exón 2 de PARK2 en un estudio realizado en una familia grande antioqueña ${ }^{14}$, lo que sugiere que en futuros proyectos de investigación es muy importante aumentar el tamaño de muestra e involucrar a todos los grupos poblacionales presentes en la geografía del país, con el objetivo de establecer un perfil genético y epidemiológico de la EPIT en esta región. En adición, se requieren estudios de expresión asociados con los cambios identificados, lo que contribuiría a un diagnóstico temprano de la enfermedad.

\section{CONCLUSIONES}

Se identificó una variante en estado homocigoto en Ala46Thr (un paciente) y en forma heterocigota en Ser167Asn (un paciente) en personas con enfermedad de Parkinson de inicio temprano; estos cambios no habían sido descritos en la población colombiana. Se desconoce si en esta población estos cambios estan relacionados con las causas y el desarrollo de la enfermedad y si el estado de homocigocidad de la primera y de heterocigocidad de la segunda en los pacientes incluidos están asociados con la severidad en las manifestaciones clínicas, por lo cual es indispensable ejecutar otro tipo estudios que permitan demostrar dicha asociación.

\section{Agradecimientos}

A Nubia Graciela Rodríguez, presidenta de la Liga Colombiana de Parkinson, por su invaluable colaboración.

\section{FinANCIACIÓN}

El proyecto fue financiado en su totalidad por la facultad de Ciencias Naturales y Matemáticas de la Universidad de Rosario, Bogotá.

\section{CONSIDERACIONES ÉtICAS}

Todos los pacientes y controles firmaron previamente un consentimiento informado avalado por el Comité de ética en Investigación (CEI) Escuela de Medicina y Ciencias de la Salud (EMCS) de la Universidad del Rosario, al igual que el proyecto adelantado por nuestro grupo de investigación (CEI-ABNo2600000186).

\section{RefERenCiAs Biblográficas}

1. Lazzarini AM, Myers RH, Zimmerman TR Jr, Mark MH, Golbe LI, Sage J, et al. A clinical genetic study of Parkinson's disease: evidence for dominant transmission. Neurology. 1994;44(3):499506.

2. Van der Merwe C, Carr J, Glanzmann B, Bardien S. Exonic rearrangements in the known Parkinson's disease-causing genes are a rare cause of the disease in South African patients. Neurosci. Lett. 2016;619:168-71.

3. García S, Suárez SS, Dávalo EM, Castillo JL. Perspectiva histórica y aspectos epidemiológicos de la enfermedad de Parkinson. Med Int Mex. 2008;24(1):28-37.

4. Parkinson J. An essay on the shaking palsy. J Neuropsychiatr. Clin. Neurosci. 2002;14(2):223-36.

5. Seirafi M, Kozlov G, Gehring K. Parkin structure and function. FEBS J. 2015;282(11):2076-88.

6. Elizondo-Cárdenas G, Déctor-Carrillo MA, Martínez-Rodríguez HR, Martínez-de Villarreal L, Esmer-Sánchez MC. Genética y la enfermedad de Parkinson: Revisión de actualidades. Med. Univer. 2011;13(51):96-100.

7. Darvish H, Movafagh A, Omrani M, Firouzabadi S, Azargashb E, Jamshidi J, et al. Detection of copy number changes in genes associated with Parkinson's disease in Iranian patients. Neurosci. Lett. 2013;551:75-8.

8. Norris K, Hao R, Chen L, Lai C, Kapur M, Shaughnessy P, et al. Convergence of Parkin, PINK1, and $\alpha$-Synuclein on Stressinduced Mitochondrial Morphological Remodeling. J. Biol. Chem. 2015;290 (22):13862-74.

9. Huttenlocher J, Stefansson H, Stacy Steinberg, Helgadottir H, Sveinbjörnsdóttir S, Riess O, et al. Heterozygote carriers for CNVs in PARK2 are at increased risk of Parkinson's disease. Hum. Mol. Genet. 2015; 24(19):5637-43.

10. Bekris LM, Mata IF, Zabetian CP. The Genetics of Parkinson Disease. J. Geriatr. Psychiatry Neurol. 2010;23(4):228-42.

11. Morris HR. Genetics of Parkinson's disease. Ann. Med. 2005;37:86-96.

12. Ambroziak W, Koziorowski D, Kinga Duszyc K, Skoczylas P, 
Chromik A, Stawek J, et al. Genomic instability in the PARK2 locus is associated with Parkinson's disease. J. Appl Genetics. 2015;56(4):51-61.

13. Gómez-Chavarín M, Roldan-Roldan G, Morales-Espinosa R, Pérez-Soto G, Torner-Aguilar C. Mecanismos fisiopatológicos involucrados en la enfermedad de Parkinson. Arch. Neurocien. (Mex. D.F.). 2012;17(1):25-33.

14. Pineda-Trujillo N, Dulcey Cepeda A, Arias Pérez W, Moreno Masmela S, Saldarriaga Henao A, Sepúlveda Falla D, et al. Una mutación en el gen PARK2 causa enfermedad de Parkinson juvenil en una extensa familia colombiana. Iatreia. 2009;22(2):122-31.

15. Peres de Morais S. Juvenile Parkinson disease caused by parkin mutations: large deletions and pathogenic mechanisms. Disertación de maestría. Facultad de Ciencias y Tecnología de la: Universidad Nova de Lisboa. 2011.

16. Biswas A, Maulik M, Das SK, Indian Genome Variation Consortium, Ray K, Ray J. Parkin polymorphisms: risk for Parkinson's disease in Indian population. Clin. Genet. 2007;72:484-6.

17. Asakawa S, Hattori N, Shimizu A, Shimizu Y, Minoshima S, Mizuno Y, et al. Analysis of eighteen deletion breakpoints in the parkin gene. Biochem. Biophys Res Commun. 2009;389:181-6.

18. De Rijk MC, Tzourio C, Breteler MM, Dartigues JF, Amaducci L, Lopez-Pousa S, et al. Prevalence of parkinsonism and Parkinson's disease in Europe: the Europarkinson Collaborative Study. European Community Concerted Action on the Epidemiology of Parkinson's Disease. J Neurol Neurosurg Psychiatry. 1997; 62(1):10-5.

19. West A, Periquet M, Lincoln S, Lücking CB, Nicholl D, Bonifati $\mathrm{V}$, et al. Complex relationship between Parkin mutations and Parkinson disease. Am J Med Genet. 2002;114(5):584-91.

20. Kitada T, Asakawa S, Hattori N, Matsumine H, Yamamura Y, Minoshima S, et al. Mutations in the parkin gene cause autosomal recessive juvenile parkinsonism. Nature. 1998;392:605-8.

21. Sanyal J, Bhaskar LVKS, Chatterjee A, Sarkar B, Chandra Ray B, Raghavendra Rao V. Single Nucleotide Polymorphisms of PARKIN gene in ten indian populations. Antrocom Online J Anthropol. 2011;7(1):135-45

22. Peng R, Gou Y, Yuan Q, Li T, Latsoudis H, Yuan G, et al.
Mutation screening and association analysis of the arkin gene in Parkinson's disease patients from South-West China. Eur. Neurol. 2003;49(2):85-9.

23. Okubadejo N, Britton A, Crews C, Akinyemi R, Hardy J, Singleton A, et al. Analysis of Nigerians with Apparently Sporadic Parkinson Disease for Mutations in LRRK2, PRKN and ATXN3. Plos One. 2008;3(10)e3421:1-4

24. Zhang Y, Wang ZZ, Sun HM. Lack of Association Between p.Ser167Asn Variant of Parkin and Parkinson's Disease: A MetaAnalysis of 15 Studies Involving 2,280 Cases and 2,459 Controls. Am J Med Genet B Neuropsychiatr Genet. 2012;159B(1):38-47.

25. Miller RJ, Wilson SM. Neurological disease: UPS stops delivering! Trends Pharmacol. Sci. 2003:24(1):18-23.

26. Polymeropoulos MH, Lavedan C, Leroy E, Ide SE, Dehejia A, Dutra $\mathrm{A}$, et al. Mutation in the alpha-synuclein gene identified in families with Parkinson's disease. Science. 1997; 276(5321):2045-7.

27. Gasser T. Genetics of Parkinson's disease. Clin Genet.1998;54(4):259-65.

28. Leroy E, Boyer R, Auburger G, Leube B, Ulm G, Mezey E, et al. The ubiquitin pathway in Parkinson's disease. Nature. 1998;395(6701):451-2.

29. Valente EM, Bentivoglio AR, Dixon PH, Ferraris A, Ialongo T, Frontali M, et al. Localization of a novel locus for autosomal recessive early-onset parkinsonism, PARK6, on human chromosome 1p35-p36. Am J Hum Genet. 2001;68(4):895-900.

30. Van Duijn CM, Dekker MC, Bonifati V, Galjaard RJ. HouwingDuistermaat JJ, Snijders PJ, et al. Park7, a novel locus for autosomal recessive early-onset parkinsonism, on chromosome 1p36. Am J Hum Genet. 2001;69(3):629-34.

31. Paisán-Ruíz C, Jain S, Evans EW, Gilks WP, Simón J, Van der Brug $\mathrm{M}$, et al. Cloning of the gene containing mutations that cause PARK8-linked Parkinson's disease. Neuron. 2004;44(4):595-600.

32. Hicks AA, Pétursson H, Jónsson T, Stefánsson H, Jóhannsdóttir HS, Sainz J, et al. A susceptibility gene for late-onset idiopathic Parkinson's disease. Ann Neurol. 2002; 52(5):549-55.

33. Pankratz N, Nichols WC, Uniacke SK, Halter C, Murrell J, Rudolph A, et al. Genome-wide linkage analysis and evidence of gene-by-gene interactions in a sample of 362 multiplex Parkinson disease families. Hum Mol Genet. 2003:12:2599-608. 